\title{
The Brazilian Portuguese version of the Exercise Adherence Rating Scale (EARS-Br) showed acceptable reliability, validity and responsiveness in chronic low back pain
}

Mariana Romano de Lira', Anamaria Siriani de Oliveira², Roberta Aniceto França ${ }^{3}$, Ana Claudia Pereira ${ }^{3}$, Emma L. Godfrey ${ }^{4,5}$ and Thais Cristina Chaves ${ }^{2^{*}}$ (D)

\begin{abstract}
Background: This study aimed to adapt the Exercise Adherence Rating Scale (EARS) into Brazilian Portuguese and evaluate its measurement properties, given as reliability, validity, and responsiveness in patients with non-specific Chronic Low Back Pain (CLBP).

Methods: A total of 108 patients with a mean age of 46.62 years $(S D=9.98)$ and CLBP participated in this longitudinal study. Participants were oriented on undertaking the prescribed exercises in the first session, and adherence behavior was assessed after 1 week, and finally reassessed after 2 weeks (test-retest reliability). Three weeks after the first assessment, they were invited again to full fill the EARS (responsiveness). The intraclass correlation coefficient $\left(\mathrm{ICC}_{2,1}\right)$ and Cronbach's a were used to assess test-retest reliability and internal consistency, respectively. Spearman's correlation and confirmatory factor analysis (CFA) were used to assess construct validity, and the Receiver operating characteristic curve and area under the curve (AUC) were used to analyze responsiveness.

Results: The one-factor EARS-Br (adherence behavior) structure with 6 items showed acceptable fit indexes (comparative fit index and goodness of fit index $>0.90$ and root-mean-square error of approximation $<0.08$ ). The EARS-Br scale showed acceptable internal consistency $(a=0.88)$ and excellent reliability $(\mathrm{ICC}=0.91[95 \% \mathrm{Cl} 0.86-$ 0.94]). Mild to moderate correlations were observed between EARS-Br total score vs. disability, pain catastrophizing, depression/anxiety, fear-avoidance and pain intensity. A Minimally Important Change (MIC) of 5.5 in the EARS-Br total score was considered as a meaningful change in the adherence behavior (AUC $=0.82$ ). Moderate accuracy (AUC $=0.89$ ) was obtained for a 17/24 total EARS cutoff score after home exercise was prescribed. The sensitivity and specificity were also acceptable (greater than $80 \%)$.

(Continued on next page)
\end{abstract}

\footnotetext{
* Correspondence: chavestc@fmrp.usp.br

${ }^{2}$ Department of Health Sciences and Post Graduate Program on

Rehabilitation and Functional Performance, Ribeirão Preto Medical School,

University of São Paulo, Ribeirão Preto, São Paulo, Brazil

Full list of author information is available at the end of the article
}

C The Author(s). 2020 Open Access This article is licensed under a Creative Commons Attribution 4.0 International License, which permits use, sharing, adaptation, distribution and reproduction in any medium or format, as long as you give appropriate credit to the original author(s) and the source, provide a link to the Creative Commons licence, and indicate if changes were made. The images or other third party material in this article are included in the article's Creative Commons licence, unless indicated otherwise in a credit line to the material. If material is not included in the article's Creative Commons licence and your intended use is not permitted by statutory regulation or exceeds the permitted use, you will need to obtain permission directly from the copyright holder. To view a copy of this licence, visit http://creativecommons.org/licenses/by/4.0/. The Creative Commons Public Domain Dedication waiver (http://creativecommons.org/publicdomain/zero/1.0/) applies to the data made available in this article, unless otherwise stated in a credit line to the data. 
(Continued from previous page)

Conclusion: Our results demonstrated acceptable EARS-Br reliability, validity, and responsiveness for patients with CLBP. A final score of 17/24 on EARS after the prescription of home-exercise could be used as a cut-off for an acceptable adherence behavior associated with improvement in patient outcomes.

Keywords: Validity studies, Chronic low Back pain, Adherence, Prescribed exercise, Responsiveness

\section{Background}

Adherence has been defined as the extent to which a person's behavior corresponds with an agreed recommendation from a health care provider [1]. It is a multidimensional construct that can be affected by factors related to the health condition, the subject (such as self-efficacy, attitudes, psychosocial factors and socioeconomic status), and the interaction between the subject and healthcare professionals [2]. As adherence is considered as a behavior, strategies that assess frequency or duration (e.g., using adherence diaries) [1] after the subject has been oriented on performing prescribed exercises cannot provide reliable insights into adherence behavior. A previous review highlighted that adherence diaries lack predictive validity for functional outcomes and that there is an urgent need to develop valid and reliable measures to assess home-prescribed exercise adherence [1].

Low back pain, which is recognized as the number one cause of global disability, had an overall point prevalence of $7.3 \%$ in 2015 , implying that 540 million people worldwide were affected [3]. Low back pain is the leading chronic health problem in the world [4]. Current guidelines encourage active treatments for patients with Chronic Low Back Pain (CLBP) [5-7] since inactivity contributes negatively to recovery [7]. This gradual shift from exercise interventions administered in clinical settings to home exercise programs [8] encourages patients to change their lifestyles, engage in models of shared decisionmaking and save on costs. However, the assessment of the adherence to prescribed home exercises is the sine qua non for investigating the relationship between engagement, dose, and effectiveness. A systematic review [9] emphasized that the majority of the studies that assess the effects of exercise interventions did not investigate adherence to exercise. Long-term adherence to home exercise programs is important for patients with CLBP to maintain lasting benefits and reduce health costs [1], given the persistence of the condition. Considering such gap in the literature, Newman-Beinart et al. [10] developed the Exercise Adherence Rating Scale (EARS), which is a brief selfreport measure comprised of three sections; the second section (B), with six items, is used to assess adherence behavior [11]. The original scale demonstrated acceptable outcomes in a population with CLBP [10].
Before a patient-reported outcome measure (PROM) is used to evaluate individuals from other countries and different cultures, it must be translated into the intended language and culturally adapted to the country in which it will be used $[12,13]$. Moreover, before use in clinical or academic contexts, the measurement properties of the adapted version of the questionnaire should be established. COnsensus-based Standards for the selection of health Measurement Instruments (COSMIN) initiative recommends that instruments should be assessed regarding measurement properties in three main domains: reliability (the degree to which the measurement is free from measurement error), validity (the degree to which a PROM measures the construct(s) it purports to measure) and responsiveness (the ability of a PROM to detect change over time in the construct to be measured).

[14].

To our knowledge, there is no validated scale for assessing adherence to prescribed exercises in Brazilian Portuguese. Therefore, the objectives of this study were to translate and culturally adapt the original version of EARS to Brazilian Portuguese and test its measurement properties (construct validity, structural validity, internal consistency, reliability and responsiveness) in patients with non-specific CLBP.

\section{Methods \\ Participants}

One-hundred and eight patients between 18 and 60 years were enrolled in this study. They were recruited through medical referrals to physiotherapy outpatient service. Patients were contacted consecutively by phone, using their waiting list, and invited to participate in this study between August 2017 and February 2019. Patient eligibility was established using the following criteria: medical diagnosis of non-specific CLBP, pain in the last three months and/or pain on at least half of the days in the last six months [15], localized pain between the last thoracic vertebra and gluteal folds and people fluent in Brazilian Portuguese. Participants with a Mini-Mental State Examination (MMSE) score below cutoff values (better educated, score $\leq 23$ and in the lower education, score $\leq 17$ ) [16], illiterate people, with degenerative systemic diseases, neurological symptoms, lumbar stenosis, spondylolisthesis, history of spinal surgeries and 
pregnancy were excluded. Written informed consent was obtained from each patient, and their rights were protected. This study was approved by the Ethics Committee Board of the Centro de Saúde Escola Cuiabá from Ribeirão Preto School of Medicine - University of São Paulo (process number: 70955617.0.0000.5414) and.

\section{Procedure}

All subjects participated in three sessions, including the following activities: Session (1) - baseline assessment (self-report questionnaires) and prescription of home exercise by a physiotherapist (motor control exercises); Session (2) - EARS administration after 1 week to investigate adherence behavior; Session (3) - retest of EARS and psychosocial reassessment - the retest was applied at a 1-week interval. Patients were contacted via telephone for responsiveness analysis, and the EARS, global perceived effect and numeric pain rating scales were reapplied three weeks after the first assessment. Figure 2 depicts a flow diagram illustrating the whole procedure.

\section{Instruments}

Exercise Adherence Rating Scale (EARS) is a self-report measure developed by a group of United Kingdom researchers [10] that is composed of six items that directly assess adherence behavior (also called as Section B). The six items are summed and items with positive phrases are reversely scored; meaning items 1,4 and 6 . The six items are scored using an ordinal answer scale $(0=$ strongly agree to $4=$ totally disagree), with higher scores indicating greater adherence (0 to 24). EARS was developed with two supporting optional sections: Section A and C. Section $C$ has 10 items related to "reasons for adherence" or non-adherence (EARS-RA). Six additional questions, which allow open answers, were developed to obtain information about the exercise recommendations (Section A).

The Pain Self-Efficacy Questionnaire (PSEQ) [17] assesses confidence in the personal ability to perform well, despite the pain. It was translated to Brazilian Portuguese and validated [18]. The PSEQ has 10 items related to the tasks frequently reported as problematic by patients with chronic pain. The items are classified with an ordinal scale from 0 to $6 ; 0=$ not confident and $6=$ totally confident. A higher score reflects a stronger belief in self-efficacy (0 to 60).

The Fear-Avoidance Beliefs Questionnaire (FABQ) adapted and validated to Brazilian Portuguese [19], is composed of 16 items, with seven answer options each, from zero (completely disagree) to 6 (completely agree). The result should be obtained separately in each of the subscales. The work-related score ranges from 0 to 42 points, and the subscale related to physical activities ranges from 0 to 24 points.
The Pain Catastrophizing Scale (PCS) is a selfadministered instrument developed to assess the degree of pain catastrophizing. It was adapted and validated for Brazilian Portuguese [20] and is composed of 13 items with answers ranging from 0 to 5 points. The patient must report the degree to which he/she recognizes any thought or feeling described by the item, and higher scores depict more severe pain catastrophizing. The instrument is subdivided into three subscales: amplification, rumination and helplessness.

The Hospital Anxiety and Depression Scale (HADS) is a self-administered scale used to identify anxiety and depression disorders in physically debilitated patients. This scale was translated and validated for Brazilian Portuguese [21]. It has two subscales, anxiety (HADS-A) and depression (HADS-D), with seven items in each domain. Each item has four response options ranging from 0 ("not at all") to 3 ("most of the time"). The score for each subscale is up to 21 points and anxiety and/or depression is depicted by scores $\geq 8$ points.

The Roland Morris Disability Questionnaire (RMDQ) assesses pain-related disability through statements related to activities of daily living. It is self-administered and has been adapted and validated for Brazilian Portuguese [22]. It has 24 items and the questionnaire score is calculated by adding the total number of questions marked with a "yes" answer. Thus, the score varies from 0 to 24 points, with 0 being the absence of disability and 24 being severe disability.

The Numeric Pain Rating Scale (NPRS) is a simple, easy-to-measure scale consisting of a sequence of integers, from 0 to 10; 0 represents "no pain" and 10 represents "worst possible pain". The measurements have acceptable levels of reliability [23].

The Global Perceived Effect (GPE) is a Likert-type, 11point scale (ranging from -5 to +5 ) that compares the patient's current condition with his or her condition at the onset of symptoms. Positive and negative scores are assigned to patients who are better and worse, respectively [23].

\section{Measurement property studies}

To make the interpretation of the results easier, all the measurement properties adopted, the methods, statistical analysis and results were described separately in different studies.

\section{Study 1 - cross-cultural adaptation of the EARS to Brazilian Portuguese and pre-testing}

Initially, we requested the permission of the author of the original scale for the cross-cultural adaptation (E. L. Godfrey). The process followed a guideline commonly used in research [12, 13]. The cross-cultural adaptation process is detailed in Fig. 1. 


\section{Study 2 - reliability and internal consistency}

Eighty-three respondents of the final version of EARS-Br were asked to complete the questionnaire again after one week, to check for test-retest reliability. The oneweek period was previously recommended [24]. For this stage, we considered only individuals with clinical stability and variations of less than 2 on the NPRS [25].

We also assessed measurement error through distribution-based methods: standard error of measurement (SEM) and minimally detectable change (MDC).

\section{Study 3- construct validity (structural validity included)}

Construct validity can be defined as the degree to which the scores of an instrument are consistent with the hypotheses and it could be obtained by comparisons with other instruments [24]. To evaluate construct validity, we assessed structural validity and conducted hypothesis testing.

The structural validity estimates the degree to which the scores of a measuring instrument are adequate reflections of the dimensionality of the construct to be measured. Although the factor structure of EARS was previously described by exploratory factor analysis (EFA) [10], we adopted confirmatory factor analysis (CFA) [26] since the better approach was to confirm the factor structure.

For the construct validity - hypothesis testing, we ran correlations between the EARS-Br score and comparator instruments scores. Relationships between test scores and other measures intended to assess the same or similar constructs provide convergent validity, whereas relationships between measures of different constructs provide discriminant validity. It is assumed that discriminant validity is established by demonstrating that convergent correlations are higher than discriminant correlations [27]. For construct validity - hypothesis testing, we formulated a priori hypotheses based on previous publications [10], as follows:

$\mathrm{H}_{1}$ ) Mild/moderate, negative, and discriminant correlations between EARS-Br scores vs. FABQ, PCS, HADS, pain intensity (NPRS), and disability (RMDQ),

$\mathrm{H}_{2}$ ) Mild/moderate, positive, and discriminant correlation between EARS-Br scores vs. PSEQ,

$\mathrm{H}_{3}$ ) Moderate to strong, positive, and convergent correlation between EARS-Br vs. EARS-RA-Br,

$\mathrm{H}_{4}$ ) Higher correlations between EARS-Br vs. EARSRA-Br than the other comparisons tested.

If $75 \%$ of the hypotheses are confirmed, construct validity is considered suitable [24].

\section{Study 4- responsiveness}

The responsiveness refers to the ability of an instrument to detect change at two different time points, or the ability of an instrument to change relative to the change of

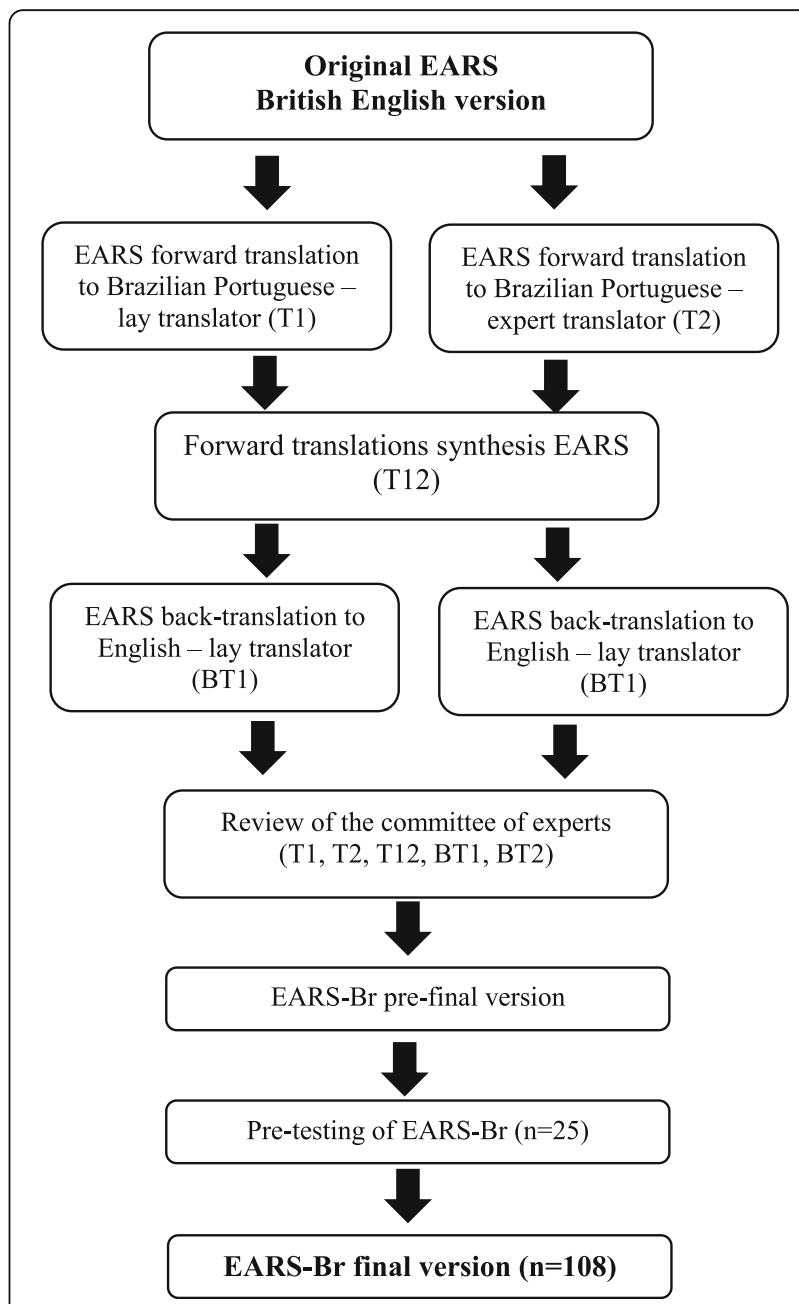

Fig. 1 Flowchart of the process of cross-cultural adaptation to Brazilian Portuguese of the Exercise Adherence Rating Scale (EARS) in five stages: I) initial translation into Brazilian Portuguese - original version in British English was translated into Portuguese by two translators fluent in English and native speakers of Portuguese - a layman and an expert in health sciences, who worked independently; II) synthesis of translations - both translations were synthesized through consensus; III) back-translation into the original language - two translators fluent in Portuguese and native English speakers back-translated the synthetized version into English. They worked independently and both were blinded to the original version; IV) specialist committee - meeting with translators $(n=4)$, physiotherapists $(n=6)$ and $\mathrm{PhDs}$ and researchers with expertise in exercise $(n=4)$ to solve possible disagreements in translation, and create a pre-final version of EARS-Br and V) pre-testing phase - in which the pre-final version of the questionnaire was administered to patients with CLBP $(n=25)$ and assessed regarding comprehensibility of the instrument controlled through an open field form and cognitive interviews. Participants were encouraged to report their possible doubts, impressions of each item, response options, header items, instructions, and instrument layout

a reference measure (external anchor) [24]. We assessed responsiveness using two construct approaches as defined by the COSMIN Study Design checklist for patient 
reported outcome measures [26]: (a) correlation between changes in scores and hypotheses testing; (b) comparison with other outcome measurement instruments.

For responsiveness based on the construct approach, we expected moderate to strong positive correlations between EARS change and GPE scores $\left(\mathrm{H}_{5}\right)$ and moderate to strong negative correlations between EARS change and pain intensity scores $\left(\mathrm{H}_{6}\right)$. We also checked for accuracy using the receiver operating characteristic curve (ROC curve) and minimally important change (MIC). The MIC is defined as the smallest change in score in the construct to be measured, which is perceived as important by patients, clinicians, or relevant others [28]. The reference measure adopted to assess MIC was the GPE (external anchor). Therefore, we raised hypotheses a priori: $\mathrm{H}_{7}$ ) moderate accuracy of EARS change score to detect who improved (increase in the score) on GPE and $\mathrm{H}_{8}$ ) moderate accuracy of EARS final score to detect who improved (reduction in the score) on GPE. A higher score on EARS-Br correlated with a higher improvement in GPE. Two units of change were deemed as an improvement on GPE [29].

\section{Statistical analysis \\ General statistics}

Analysis of Variance (ANOVA) was performed to test for significant differences between subsamples of the different studies included in the project $(p<0.05)$. All analyses were performed using the SPSS statistical package for Windows and IBM SPSS, version 22.

\section{Study 2 - reliability and internal consistency}

Reliability was calculated using the intraclass correlation coefficient $\left(\mathrm{ICC}_{2,1}\right.$, two-way random effect model). ICC values were classified as poor $(<0.40)$, moderate $(0.40-$ $0.75)$ and excellent $(>0.75)$ [30]. We calculated the SEM and MDC [31]. MDC is the smallest change that can be detected by the instrument beyond measurement error [24]. Both MDC and MIC (see below on study 4) should be higher than SEM [24].

The standard error of measurement (SEM) was analyzed using the following formula: $S E M=S D \times \sqrt{ }(1-$ ICC), in which $\mathrm{SD}=$ standard deviation.

The MDC is considered a distribution-based measure and was calculated as follows: $\mathrm{MDC}_{95}=1.96 \times \sqrt{2} \times$ SEM.

The internal consistency was analyzed using Cronbach's $\alpha$ with acceptable results between 0.70 and 0.95 [24].

\section{Study 3 - construct validity}

To check for the structural validity of the EARS-Br scales, CFA was used. We analyzed the goodness of fit of three models: i) EARS-Br with a one-factor model with 6 items [8]; ii) One-factor EARS-RA-Br model with 10 items and iii) One-factor EARS-RA-Br with 9 items (item 8 was excluded). We investigated the factoriability of the dataset using an EFA approach assessing the following measures: Kaiser-Meyer-Olkin (KMO) with acceptable values ranging from 0.5 to 1 [32] and Bartlett's test of sphericity, for which a cut-off below of 0.05 is recommended [33].

IBM SPSS AMOS (version 22) was used to run the CFA. As we identified a violation of multivariate normality, we run the analysis using a bootstrap maximum likelihood (ML) method (2000 resamples) [34]. Bollen-Stine gauges fit without normal theory limitations [35], and $p>0.05$ suggests the acceptance of the null hypothesis of global fit (the model is correct).

Acceptability of fit was evaluated based on several indexes: root mean square error of approximation (RMSEA, recommended value below 0.08), comparative fit and goodness of fit indexes (CFI and GFI, recommended value close to 0.90), Expected Cross-Validation index and Consistent Akaike Information Criterion (ECVI and CAIC - lower values, best fit [36]), and CMIN/df (degrees of freedom) - should be less than 3 [36]. The magnitudes of factor loadings of 0.3 or greater [37] were considered suitable.

To assess for construct validity, hypothesis testing and spearman's rho were used, and coefficients above 0.7 were classified as strong, those between 0.69 and 0.3 as moderate, and those below 0.29 as mild/weak [38].

\section{Study 4 - responsiveness}

We adopted three analyses to check for responsiveness: (a) correlation between change scores (construct approach, hypotheses testing - comparison with other outcome measurement instruments), (b) Determining the MIC for EARS-Br anchor-based responsiveness and (c) Determining the cut-off score for EARS-Br.

\section{Correlation between change scores (construct validity - hypotheses testing)}

We calculated the correlation between mean changes in scores for EARS vs. GPE and EARS vs. pain intensity using the Spearman rank correlation. The same classification for grading the magnitude of correlation was used, as described above [38].

\section{Determining the MIC for EARS-Br}

The MIC should be measured using an anchor-based approach in which an external anchor is adopted to run comparisons. We used GPE in the current study. We adopted the following metric to obtain the change in

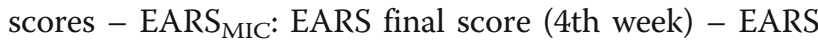
initial score (2nd week). 
To calculate MIC, receiver operating characteristic (ROC) curves were plotted showing sensitivity and 1specificity values and area under the curve (AUC) showing the probability of correctly discriminating between patients who improved (a change of at least 2 units as a criterion for improvement) and worsened/remained stable according to GPE (reference measure). The MIC for EARS was determined as the point of optimal cutoff in ROC curves related to greater sensitivity and specificity values [39], and higher than MDC [24].

\section{Determining the cut-off score for $E A R S-B r$}

Beyond the MIC calculation, the EARS was used after the completion of the home exercise programs to assess adherence behavior retrospectively. A cut-off for the EARS score was also determined to guide the interpretability of EARS results. It was obtained by determining the minimum final EARS cut-off score of adherence behavior with a score of at least 2 units of improvement on GPE. The AUC classification used was: ROC $>0.9$ : high accuracy, $0.7<$ ROC $<0.9$ : moderate accuracy, $0.5<$ ROC $<0.7$ : low accuracy and ROC $<0.5$ : chance [39].

\section{Results}

\section{Overall findings}

Initially, 145 patients were invited to participate in this study and 37 were excluded because they did not meet the eligibility criteria. The final sample included 108 individuals. The pre-testing sample was comprised of 25 patients. For the test-retest reliability, we enrolled 76 participants (invited from the initial 108 participants) who had pain intensity changes less than 1 unit during the one week between baseline and test-retest assessments. Eighty-three patients with CLBP were assessed for responsiveness (Fig. 2). The clinical, educational and anthropometric data of the different subsamples of the studies are described in Table 1. A significant difference between subsamples considered in the distinct steps of the current study was observed only for the EARS-RA$\mathrm{Br}$ score and the total score of the MMSE. However, for cognitive evaluation, all volunteers showed a cutoff value above the minimum for normal cognitive level [16].

\section{Study 1 - cross-cultural adaptation of EARS-Br and pre- testing}

During the meetings for cross-cultural translation and adaptation, there was a consensus on most of the questions among the members of the translation committee. However, the committee did not agree on one item of the EARS and two items of the EARS-RA. After a conversation with the author of the original version, the items were translated as below:
1. "I don't get around to doing my exercises" - Eu não consigo me organizar para fazer os meus exercícios (the target meaning should be "cannot organize to do exercises")

2. "I feel confident about doing my exercises" - Eu sinto autoconfiança para fazer os meus exercícios (the target meaning should be self-efficacy to exercise)

3. "I stop exercising when my pain is worse" - Eu interrompo o exercício quando minha dor piora (the target meaning should discontinue exercise when the pain gets worse, rather than to usually avoid exercising when the pain gets worse).

Moreover, the committee suggested the inclusion of descriptions for all possible response options and the authors of the original scale agreed with that adaptation. During the pre-testing, no volunteer reported any type of difficulty and/or suggestions for the EARS-Br.

The full questionnaire is available as a Supplementary File.

\section{Study 2 - reliability and internal consistency}

Table 2 describes reliability, SEM, and MDC. For EARS-Br, the Cronbach's $\alpha$ was acceptable (0.88). The ICC values for the EARS-Br scores were considered excellent (Table 2).

\section{Study 3 - construct validity}

The EFA showed acceptable KMO values for EARS-Br and EARS-RA-Br (0.86 and 0.64), and Bartlett index $(p<0.001)$. Afterward, we investigated the fit of three different models as described in the statistical analysis. After the application of the bootstrap ML method, the Bollen-Stine $p$-value for the EARS-Br and EARS-RA-Br showed acceptable values. An acceptable fit was also observed for the EARS-Br with 6 items (Table 3). The factor loadings for both scales are depicted in Fig. 3. The EARS-RA-Br with 9 items also showed acceptable fit indexes (Table 3). Item 8 (I adjust the way I do my exercises to suit myself) was removed from the scale for "reasons for adherence" to exclusion improve the indices of fit (Table 3). Item 5 showed a poor factor loading (0.26), however, it was not excluded because it did not impair the overall fit of the scale (Table 3).

Correlations between the EARS-Br scores and psychosocial scales are described in Table 4 . We confirmed the hypotheses raised a priori $\left(\mathrm{H}_{1}, \mathrm{H}_{3}, \mathrm{H}_{4}\right)$, except for the correlation between PSEQ score and EARS- $\mathrm{Br}\left(\mathrm{H}_{2}\right)$ (Table 4).

\section{Study 4 - responsiveness \\ Correlation between change scores - construct approach, hypotheses testing}

There was a moderate positive correlation in mean changes of scores between EARS-Br and GPE ( $r=0.65$, 


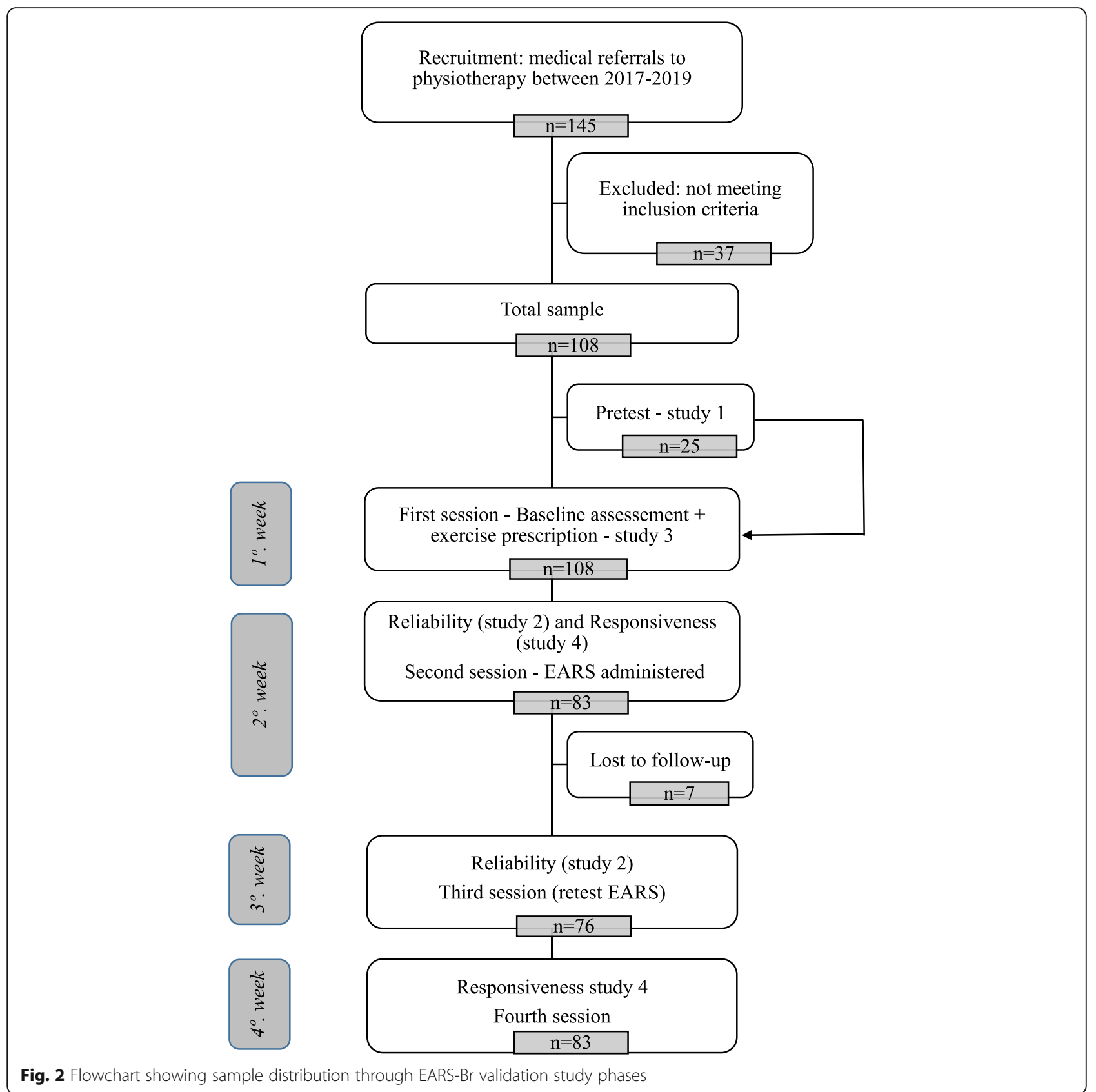

$p<0.001)$, and a moderate negative correlation between EARS-Br and pain intensity (NPRS) $(\mathrm{r}=-0.58, \mathrm{p}<$ 0.001). Hence, we confirmed our hypotheses $\mathrm{H}_{5}$ and $\mathrm{H}_{6}$.

\section{Determining the MIC for EARS-Br}

The responsiveness analysis showed moderate accuracy (AUC $=0.82)$ for a MIC of 5.5 (decrease) on EARS-Br, in distinguishing between patients that got worse or stable $(n=27)$ and those who improved $(n=57)$, considering the GPE as the reference measure (confirming $\mathrm{H}_{7}$ ). We showed a $93 \%$ sensitivity to detect those who reported worsening/stability and a $48 \%$ specificity to detect those who improved for the MIC of 5.5 (Table 5, Fig. 4a).

\section{Determining the cut-off score for EARS-Br}

We also found a moderate accuracy $(A U C=0.89)$ for the cut-off of $17 / 24$ on EARS-Br to distinguish between patients who improved $(n=57)$ and those who got worse or stable $(n=27)$ when considering GPE as the reference measure (confirming $\mathrm{H}_{8}$ ). We showed a sensitivity (ability to detect who improved on GPE) and specificity (ability to detect who got worse or stable) higher than $80 \%$ for the cut-off EARS score of 17 (Table 5, Fig. 4b). 
Table 1 Description (mean and standard deviation: SD) of anthropometric, schooling and clinical/ psychosocial data of patients recruited in this study $(n=108)$

\begin{tabular}{|c|c|c|c|c|c|}
\hline \multicolumn{6}{|l|}{ Sample } \\
\hline & $\begin{array}{l}\text { Pre-testing sample Mean } \\
\text { (SD) }\end{array}$ & $\begin{array}{l}\text { Reliability sample Mean } \\
\text { (SD) }\end{array}$ & $\begin{array}{l}\text { Responsiveness sample } \\
\text { Mean (SD) }\end{array}$ & $\begin{array}{l}\text { Validity sample Mean } \\
\text { (SD) }\end{array}$ & $\begin{array}{l}\text { ANOVA }\left(F_{(3,288)}\right. \\
\text { p) }\end{array}$ \\
\hline Sample Size & 25 & 76 & 83 & 108 & \\
\hline Age & $46.33(10.51)$ & $46.84(10.06)$ & $46.71(9.89)$ & $46.62(9.98)$ & $0.01, p=0.98$ \\
\hline Weight & $80.72(19.44)$ & $77.33(14.93)$ & $77.77(14.84)$ & $78.47(16.01)$ & $0.32, p=0.72$ \\
\hline Height & $1.68(0.10)$ & $1.67(0.08)$ & $1.66(0.08)$ & $1.68(0.09)$ & $0.29 p=0.74$ \\
\hline Pain intensity & $3.52(2.27)$ & $4.08(2.91)$ & $4.08(2.87)$ & $3.95(2.74)$ & $0.40, p=0.66$ \\
\hline Years lived with pain & $7.80(5.77)$ & $6.06(6.47)$ & $6.06(6.54)$ & $6.74(6.48)$ & $0.71, p=0.49$ \\
\hline Female prevalence (\%) & $64 \%(n=16)$ & $67 \%(n=52)$ & $55 \%(n=45)$ & $57 \%(n=61)$ & $0.28, p=0.75$ \\
\hline Level of education ${ }^{\mathrm{a}}$ & $1.6(0.57)$ & $1.69(0.73)$ & $1.6(0.72)$ & $1.6(0.69)$ & $0.19, p=0.82$ \\
\hline \multicolumn{6}{|l|}{ Mean score (SD) } \\
\hline EARS & $16.28(6.32)$ & $17.91(6.71)$ & $17.75(6.62)$ & $17.41(6.55)$ & $0.48, p=0.61$ \\
\hline EARS-RA & $23.36(7.39)$ & $25.14(6.41)$ & $27.45(6.67)$ & $26.50(7.03)$ & $3.34, p=0.03^{*}$ \\
\hline HADS anxiety (0-21) & $9.64(4.07)$ & $7.34(3.97)$ & $8.39(3.71)$ & $9.10(3.79)$ & $0.32, p=0.72$ \\
\hline $\begin{array}{l}\text { HADS depression } \\
(0-21)\end{array}$ & $6.60(4.18)$ & $5.36(4.00)$ & $6.66(3.78)$ & $6.64(3.79)$ & $0.00, p=0.99$ \\
\hline PESQ (0-60) & $36.64(17.57)$ & $44.75(13.58)$ & $40.13(15.27)$ & $39.32(15.81)$ & $0.46, p=0.62$ \\
\hline $\begin{array}{l}\text { PCS magnification } \\
(0-12)\end{array}$ & $5.40(3.13)$ & $4.68(3.31)$ & $4.76(3.32)$ & $4.91(3.27)$ & $0.07, p=0.92$ \\
\hline $\begin{array}{l}\text { PCS rumination }(0- \\
16)\end{array}$ & $7.56(3.90)$ & $7.57(3.44)$ & $7.66(3.51)$ & $7.64(3.58)$ & $0.03, p=0.97$ \\
\hline $\begin{array}{l}\text { PCS helplessness (0- } \\
\text { 24) }\end{array}$ & $8.36(6.05)$ & $8.48(5.96)$ & $8.47(5.98)$ & $8.44(5.96)$ & $0.13, p=0.87$ \\
\hline FABQ phys (0-24) & $12.56(6.70)$ & $10.73(6.96)$ & $14.30(6.79)$ & $13.89(3.78)$ & $0.63, p=0.53$ \\
\hline FABQ work (0-42) & $27.8(12.35)$ & $21.77(13.09)$ & $25.04(13.61)$ & $25.68(13.32)$ & $0.40, p=0.66$ \\
\hline RMDQ (0-24) & $12.8(6.29)$ & $11.69(5.88)$ & $13.42(5.36)$ & $13.27(5.57)$ & $0.11, p=0.88$ \\
\hline
\end{tabular}

aLevel of education: 1 = Incomplete/complete basic education; 2 = Incomplete/complete high school; $3=$ Incomplete/complete higher education EARS = Exercise Adherence Rating Scale; EARS-RA = Exercise Adherence Rating Scale, reasons for adherence; HADS = Hospital Anxiety and Depression Scale; $\mathrm{PESQ}=$ Pain Self-Efficacy Questionnaire; PCS = Pain Catastrophizing Scale; FABQ = Fear Avoidance Beliefs Questionnaire; RMDQ = Roland Morris Disability Questionnaire; MMSE = Mini-Mental State Examination

$\mathrm{n}=$ sample size. ${ }^{*} p<0.05$

\section{Discussion}

This study carried out the cross-cultural adaptation of the EARS [8] for Brazilian Portuguese in patients with CLBP following international recommendations $[13,26]$. EARS-Br showed excellent acceptability and comprehension during pre-testing and psychometric analyses. It also demonstrated acceptable reliability, internal consistency, construct and structural validity, and responsiveness. It is the first valid PROM available in Brazilian Portuguese that evaluates behavior adherence to prescribed exercises in patients with CLBP.

\section{Study 2 - reliability and internal consistency}

The test-retest reliability of the EARS-Br scores was considered excellent for both EARS-Br scales, and our findings are supported by the results of the original version of the scale [10]. The SEM and MDC values for EARS$\mathrm{Br}$ were 1.97 and 5.45, respectively. For the original
EARS, such values were not described. The MDC obtained in our study showed that any MIC for the EARS$\mathrm{Br}$ scores should be higher than 5.45 to surpass the measurement error.

Additionally, our findings showed an acceptable internal consistency (Cronbach's $\alpha>0.80$ ) for EARS-Br, which is consistent with the internal consistency results reported by the original 6 -item EARS $(\alpha=0.81)$ [10]. We did not test the internal consistency of the EARS-RA-Br because the recommendation was against adding up its items to obtain a final score [10].

\section{Study 3 - construct validity}

CFA confirmed the structure (structural validity) reported for the original EARS with 6 items, and we also checked for the structure of the EARS-RA. We showed an acceptable fit for that scale with 9 items. For EARS$\mathrm{RA}-\mathrm{Br}$, two items showed factor loadings below 0.30 . 


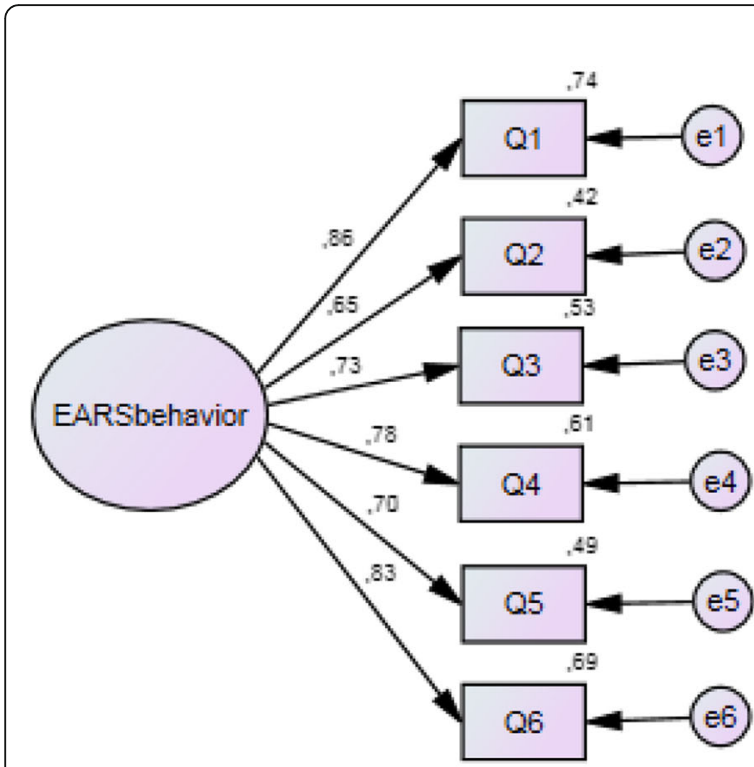

A

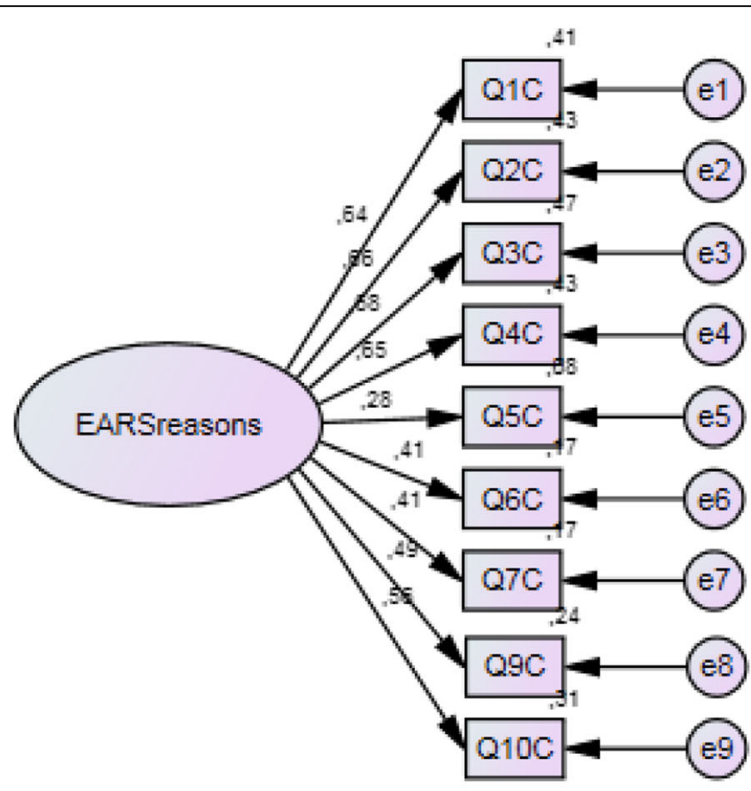

B

Figs. 3A and 3B Path diagram showing factor structure of the EARS-Br (Fig. A) and EARS-Br reasons for adherence (Fig. B) describing the factor loadings for each item. $Q=$ questions. $e=$ error. EARSbehavior $=E A R S$. EARSreasons $=$ EARS reasons for adherence or EARS-RA

Despite the poor factor loading for both items, we decided to remove only the item that impaired the scale fit (item 8). That item was the only one that did not show a correlation with the EARS-Br total score as reported in the manuscript of the original version [10]. We cannot compare our results with the findings reported for the original EARS-RA, because it was not submitted to structural validity analysis. As recommended in the original manuscript, the items of EARS-RA should not be added up to obtain a total score. However, it is recommended that items are analyzed separately to determine which specific factors significantly influence adherence behavior.

For construct validity, we hypothesized a mild to moderate correlation for the psychosocial questionnaires administered (discriminant validity), and we observed that higher scores on fear-avoidance and pain intensity lowered the scores on EARS-Br. We also showed that higher scores for anxiety, depression, disability, fear-avoidance and pain catastrophizing lowered scores on EARS-Br. In agreement with our findings, a systematic review that identified barriers to adherence to treatment in

Table 2 Minimum and maximum score for EARS-Br items, mean score values, intraclass correlation coefficient (ICC), standard error of measurement (SEM) and minimally detectable change (MDC) of each question and total score of domains of the Brazilian Portuguese version of the Exercise Adherence Rating Scale (EARS-Br) $(n=108)$

\begin{tabular}{|c|c|c|c|c|}
\hline Items & $\begin{array}{l}\text { ICC }(95 \% \mathrm{Cl}) \\
n=83\end{array}$ & $\begin{array}{l}\text { SEM/ } \\
\text { MDC } \\
n=108\end{array}$ & $\begin{array}{l}\text { Mean Value EARS-Br } \\
\text { (SD) }\end{array}$ & $\begin{array}{l}\text { Minimum-maximum Value } \\
\text { EARS-Br }\end{array}$ \\
\hline 1. I do my exercises as often as recommended & $0.87(0.80-0.92)$ & $0.45 / 1.25$ & $3.13(\mathrm{SD}=1.25)$ & $0-4$ \\
\hline 2. I forget to do my exercises & $0.80(0.69-0.87)$ & $0.60 / 1.66$ & $2.54(\mathrm{SD}=1.34)$ & $0-4$ \\
\hline $\begin{array}{l}\text { 3. I do less exercise than recommended by my healthcare } \\
\text { professional }\end{array}$ & $0.92(0.88-0.95)$ & $0.47 / 1.30$ & $2.83(\mathrm{SD}=1.56)$ & $0-4$ \\
\hline 4. I fit my exercises into my regular routine & $0.86(0.78-0.91)$ & $0.49 / 1.36$ & $3.42(S D=1.26)$ & $0-4$ \\
\hline 5. I don't get around to doing my exercises & $0.91(0.87-0.94)$ & $0.41 / 1.13$ & $3.25(\mathrm{SD}=1.44)$ & $0-4$ \\
\hline 6. I do most, or all, of my exercises & $0.89(0.83-0.93)$ & $0.43 / 1.20$ & $3.58(\mathrm{SD}=1.30)$ & $0-4$ \\
\hline Total score & $\begin{array}{l}0.91(0.86- \\
0.94)\end{array}$ & $\begin{array}{l}1.97 / \\
5.45\end{array}$ & $18.75(S D=6.56)$ & $0-24$ \\
\hline
\end{tabular}

$\mathrm{n}=$ sample size; $\mathrm{Cl}=$ confidence interval $95 \% ; \mathrm{SD}=$ Standard Deviation SEM $=$ SD $\sqrt{ } 1-$ ICC

MDC95 $=1.96 \times \mathrm{SEM} \times \sqrt{2}$ 
Table 3 Confirmatory factor analysis indices obtained for the Brazilian Portuguese version of the Exercise Adherence Rating Scale (EARS-Br) and for the EARS reasons for adherence (EARS-RA-Br) $(n=108)$

\begin{tabular}{|c|c|c|c|c|c|c|}
\hline & $\mathrm{CMIN} / \mathrm{df}$ & CAIC & $\mathrm{CFI}$ & GFI & ECVI $(90 \%$ Cl) & RMSEA ( $90 \% \mathrm{Cl})$ \\
\hline Models & EARS-Br & & & & & \\
\hline \multirow[t]{2}{*}{ EARS-Br - 6 items ${ }^{\#}$} & 1.89 & 70.14 & 0.97 & 0.93 & $0.38(0.27-0.55)$ & $0.08(0.03-0.14)$ \\
\hline & EARS-RA-Br & & & & & \\
\hline EARS-RA-Br 10 items $^{\&}$ & 1.63 & 182.95 & 0.90 & 0.91 & $0.92(0.77-1.13)$ & $0.07(0.03-0.11)$ \\
\hline EARS-RA-Br 9 items ${ }^{\& \&}$ & 1.61 & 157.97 & 0.93 & 0.93 & $0.75(0.62-0.94)$ & $0.07(0.02-0.12)$ \\
\hline
\end{tabular}

\#One factor structure with 6 items relating to adherence behavior (6-items of section B) (Beinart et al., 2016)

\&One factor structure with 10 questions relating to reasons for adherence (10-items of section C) of original EARS (Newman-Beinart et al., 2016)

${ }^{\& \&}$ One factor structure with 10 questions relating to reasons for adherence (10-items of section C) of original EARS (Newman-Beinart et al., 2016) with the exclusion of item 8

$\mathrm{CMIN} / \mathrm{df}=\mathrm{x}^{2} / \mathrm{df} ; \mathrm{CAIC}=$ consistent Akaike information criterion; RMSEA = root-mean-square error of approximation; $90 \% \mathrm{Cl}=90 \%$ confidence interval for RMSEA; $\mathrm{CFI}=$ comparative fit index; $\mathrm{GFI}=$ goodness of fit; $\mathrm{ECVI}=$ expected cross-validation index

$\mathrm{n}=$ sample size

physiotherapy outpatients showed that pain intensity, depression and anxiety were identified as barriers to adherence to exercise [40].

In this study, a correlation between pain self-efficacy and adherence behavior was not observed. Several studies have shown that poor self-efficacy could explain a patient's low confidence in their ability to overcome obstacles to initiating, maintaining or resuming from relapses in exercises [41]. On the other hand, there is no question specifically related to exercise on PSEQ, since PSEQ is a questionnaire focused on pain self-efficacy and not exercise self-efficacy. This may explain our results since self-efficacy is a task-specific construct [42]. A new instrument has been described in literature and is

Table 4 Correlations between EARS-Br vs. anxiety, depression, pain self-efficacy, fear of movement, disability, pain intensity, and reasons for adherence $(n=108)$

\begin{tabular}{ll}
\hline Questionnaires and Domains (scores) & EARS-Br \\
\hline HADS - Anxiety (0-21) & $-0.22^{*}$ \\
HADS - Depression (0-21) & $-0.25^{*}$ \\
PSEQ (0-60) & 0.16 \\
PCS- Helplessness (0-24) & $-0.22^{*}$ \\
PCS- Magnification (0-12) & $-0.22^{*}$ \\
PCS- Rumination (0-16) & $-0.27^{*}$ \\
FABQ-Phys (0-24) & $-0.37^{* *}$ \\
FABQ-Work (0-42) & $-0.21^{*}$ \\
RMDQ (0-24) & $-0.22^{*}$ \\
NPRS (0-10) & $-0.52^{* *}$ \\
EARS-RA-Br & $0.86^{* *}$ \\
\hline
\end{tabular}

${ }^{*} p<0.05$, Spearman's correlation

${ }^{* *} p<0.01$, Spearman's correlation

EARS $=$ Exercise Adherence Rating Scale; HADS $=$ Hospital Anxiety and Depression Scale; PESQ = Pain Self-Efficacy Questionnaire; PCS = Pain Catastrophizing Scale; FABQ = Fear Avoidance Beliefs Questionnaire; RMDQ = Roland Morris Disability Questionnaire; NPRS = Numerical Rating Scale; EARS$\mathrm{RA}-\mathrm{Br}=\mathrm{EARS}$ reasons for adherence

$\mathrm{n}=$ sample size available for specifically assessing self-efficacy for home prescribed exercises [43]. Future studies correlating exercise self-efficacy and adherence behavior (EARS) are therefore recommended.

We found a moderate and negative correlation between pain intensity and EARS-Br (discriminant validity). A greater intensity of pain correlated with a lower exercise adherence score. The original EARS study [10] also reported a moderate correlation between pain intensity and adherence. This may be related to the strong common patient belief that pain is a marker of tissue damage [44] and that exercise/movement may aggravate tissue damage and, consequently, pain. In a previous systematic review [40], pain intensity was also identified as a barrier to adherence, which is consistent with our findings. These results suggest that patients with higher levels of pain intensity may demonstrate worse adherence in clinical trials.

Ultimately, we found that correlations were higher between EARS-Br (adherence behavior) and EARS-RA-Br than between EARS-Br and the remaining constructs. Since both scales assess complementary aspects of exercise adherence, we consider these findings as parameters of discriminant validity [27].

\section{Study 4 - responsiveness}

EARS is an instrument to be administered after the prescription of exercises and not as a tool to assess pre- and post-intervention change. Hence, a greater EARS score at the end of the exercise program correlated with better adherence to prescribed exercise protocols. However, EARS can be used to longitudinally monitor patient adherence to exercise, and it is important to define a minimum parameter of score fluctuation when following patients prescribed with home exercises. We controlled the change in EARS scores (after home exercise prescription) and its relationship with perceived improvement in two time points. Our findings showed that an acceptable fluctuation in EARS total scores should not 
Table 5 Accuracy, cutoff, sensitivity and specificity values for the score of change (Minimally Important Change) and cut-off score for Exercise Adherence Rating Scale - Brazilian Portuguese (EARS-Br) in relation to Global Perceived Effect (GPE) $(n=83)$

\begin{tabular}{llllll}
\hline & Patients that improved & AUC (C.I. 95\%) & Cutoff & Sensitivity & Specificity \\
\hline EARS-Br (change score) vs GPE & $56(22.48, \mathrm{SD}=3.07)$ & $0.82(0.73-0.91)$ & 5.5 & 0.93 & 0.48 \\
EARS-Br (cutoff score) vs GPE & $56(22.48, \mathrm{SD}=3.07)$ & $0.89(0.79-0.98)$ & 17.5 & 0.82 & 0.89 \\
\hline
\end{tabular}

$\mathrm{ROC}>0.9$ : high accuracy, $0.7<\mathrm{ROC}<0.9$ : moderate accuracy, $0.5<\mathrm{ROC}<0.7$ : low accuracy and $\mathrm{ROC}<0.5$ : random outcome $\mathrm{AUC}=$ Area Under the Curve

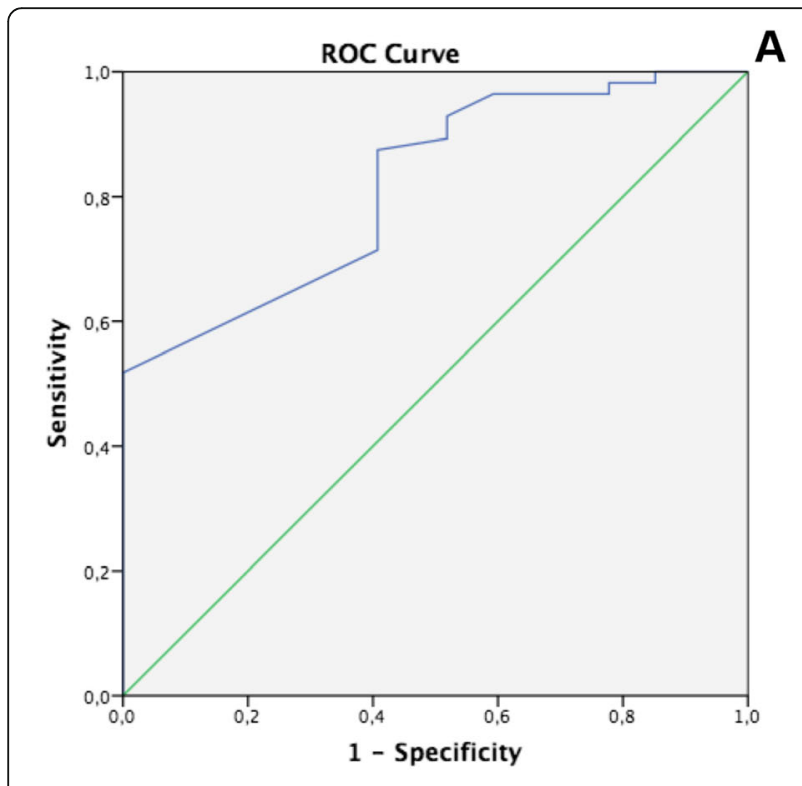

B

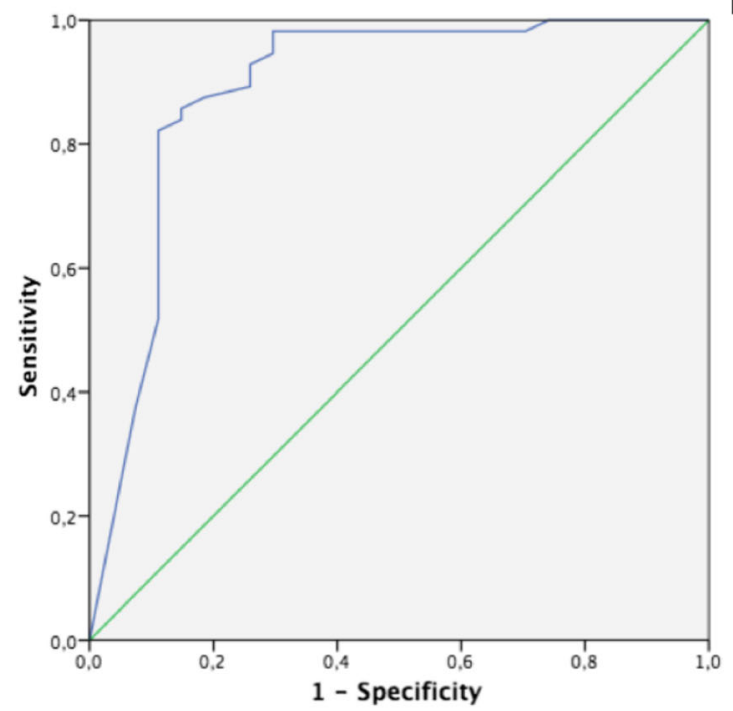

Fig. 4 Receiver Operating Curves (ROC) describing sensitivity and specificity values for responsiveness analysis of the Brazilian version of the Exercise Adherence Rating Scale (EARS-Br). A: Minimally Important Change for EARS-Br considering as reference Global Perceived Effect (GPE), comparing EARS score longitudinally. B. Cutoff score for EARS-Br for the final assessment after the exercise program considering as reference Global Perceived Effect (GPE) exceed 5.5 (MIC). Therefore, any decrease in the total EARS score greater than 5.5 during the follow-up assessments should be interpreted as a meaningful decrease in adherence behavior to home exercises, and health professionals should intervene and identify the motivations for poor adherence. The MIC for EARS showed an excellent sensitivity for detecting patients who did not report improvement on GPE (93\%), although it showed a low specificity for detecting those who improved (48\%). This suggests that EARS scores may be better for evaluating the non-adherence behavior associated with a poor perception of improvement.

Additionally, we observed that the cut-off score of 17 distinguished between patients that perceived an improvement greater than 2 units on GPE. Our results suggest that the acceptable total score should be at least 17/ 24 considering the GPE score as a reference, and we recommend this as a guide for controlling adherence behavior. It was not possible to draw comparisons between the responsiveness outcomes of our study and the original scale, as we did not perform the required analysis.

\section{Limitations}

Our study validated EARS-Br in a population with CLBP, hence the extrapolation of our results to other populations should be made with caution. It may also be valuable to investigate the validity of the EARS concurrently with an objective activity device, due to self-report bias when considering interventions that permit control for step counting (pedometers). However, there is no objective measure of adherence available for interventions used in physical therapy settings. Finally, we assessed for responsiveness during a short period of three weeks and using a small sample size. We suggest additional studies to investigate responsiveness during longer periods between assessments and using bigger sample sizes.

\section{Strength and clinical implications}

The EARS-Br is the first validated tool in BrazilianPortuguese that can assess adherence to prescribed home exercises in patients with CLBP. The scale showed acceptable measurement properties and can be used in clinical practice to follow-up on patients prescribed with home exercise programs. It can be adopted or used to 
monitor exercise adherence levels after hospital/outpatient discharge. A total EARS cut-off score of 17/24 could be used as a parameter of acceptable adherence behavior. Additionally, any decrease of 5.5 or more in the total EARS score could be adopted as a meaningful decrease in exercise adherence.

\section{Conclusion}

The EARS scales were cross-culturally adapted for Brazilian Portuguese following international recommendations. EARS-Br is a reliable and valid instrument to assess adherence to prescribed home exercises in patients with CLBP. A final score of $17 / 24$ on EARS after the prescription of home-exercise could be used as a cut-off for acceptable adherence behavior associated with improvement in patient outcomes.

\section{Supplementary information}

Supplementary information accompanies this paper at https://doi.org/10. 1186/s12891-020-03308-z.

Additional file 1. The full version Exercise Adherence Rating Scale in

Brazilian Portuguese is available as Supplementary File

\begin{abstract}
Abbreviations
CLBP: Chronic low back pain; EARS: Exercise Adherence Rating Scale; PROM: Patient reported outcome measure; COSMIN: COnsensus-based Standards for the selection of health Measurement Instruments; MMSE: MiniMental State Examination; EARS-RA: Exercise Adherence Rating Scale reasons for adherence or non-adherence; PSEQ: Pain Self-Efficacy Questionnaire; FABQ: Fear-Avoidance Beliefs Questionnaire; PCS: Pain Catastrophizing Scale; HADS: Hospital Anxiety and Depression Scale; HADS-A: Hospital Anxiety and Depression Scale - anxiety; HADS-D: Hospital Anxiety and Depression Scale - depression; RMDQ: Roland Morris Disability Questionnaire; NPRS: Numeric Pain Rating Scale; GPE: Global Perceived Effect; EFA: Exploratory Factor Analysis; CFA: Confirmatory actor analysis; ROC Curve: Receiver operating characteristic curve; MIC: Minimally Important Change; MDC: Minimally Detectable Change; ICC: Intraclass Correlation Coefficient; SEM: Standard Error of Measurement; KMO: Kaiser-Meyer-Olkin; ML: Maximum Likelihood; RMSEA: Root mean square error of approximation; CFI: Comparative fit; GFI: Goodness of fit indexes; ECVI: Expected Cross Validation Index; CAIC: Consistent Akaike Information Criterion; ROC: Receiver Operating Characteristic; AUC: Area Under the Curve; ANOVA: Analysis of Variance
\end{abstract}

\section{Acknowledgments}

Not Applicable.

\section{Authors' contributions}

TCC and MRL analyzed, interpreted the data and written the paper. MRL, RAF and ACP participated in data collection. ASO and ELG reviewed the paper. All authors read and approved the final manuscript.

\section{Funding}

This work was supported by Assistência do Hospital das Clínicas da Faculdade de Medicina de Ribeirão Preto da Universidade de São Paulo (FAEPA).

\section{Availability of data and materials}

The datasets used and/or analyzed during the current study are available from the corresponding author on reasonable request.

\section{Ethics approval and consent to participate}

The present study was approved by the local ethics committee of the Centro de Saúde Escola Cuiabá from Ribeirão Preto School of Medicine University of São Paulo (process number: 70955617.0.0000.5414). A written informed consent was taken from each patient.

\section{Consent for publication}

Not Applicable.

\section{Competing interests}

Not Applicable.

\section{Author details}

${ }^{1}$ Post Graduate Program on Rehabilitation and Functional Performance, Ribeirão Preto Medical School, University of São Paulo, Ribeirão Preto, São Paulo, Brazil. ${ }^{2}$ Department of Health Sciences and Post Graduate Program on Rehabilitation and Functional Performance, Ribeirão Preto Medical School, University of São Paulo, Ribeirão Preto, São Paulo, Brazil. ${ }^{3}$ Physical Therapy undergraduate course from Ribeirão Preto Medical School, University of São Paulo, Ribeirão Preto, São Paulo, Brazil. ${ }^{4}$ Department of Population Health Sciences, Faculty of Life Sciences \& Medicine, King's College London, 2nd Floor Addison House, Guy's Campus, London SE1 1UL, UK. ${ }^{5}$ Department of Psychology, IoPPN, King's College London, 5th Floor Bermondsey Wing, Guy's Campus, London SE1 9RT, UK.

Received: 26 January 2020 Accepted: 22 April 2020

Published online: 12 May 2020

\section{References}

1. Frost R, Levati S, McClurg D, Brady M, Williams B. What Adherence Measures Should Be Used in Trials of Home-Based Rehabilitation Interventions? A Systematic Review of the Validity, Reliability, and Acceptability of Measures. Arch Phys Med Rehabil. 2017; 98(6):1241-1256.e45.

2. Bollen JC, Dean SG, Siegert RJ, Howe TE, Goodwin V. A systematic review of measures of self-reported adherence to unsupervised home-based rehabilitation exercise programmes, and their psychometric properties. BMJ Open. 2014;4:e005044.

3. Hartvigsen J, Hancock MJ, Kongsted A, Louw Q, Ferreira ML, Genevay S, Hoy D, Karppinen J, Pransky G, Sieper J, Smeets RJ, Underwood M; Lancet Low Back Pain Series Working Group. What low back pain is and why we need to pay attention. Lancet. 2018, 9;391(10137):2356-2367.

4. Maher C, Underwood M, Buchbinder R. Non-specific low back pain. Lancet. 2017, 18;389(10070):736-747.

5. Michaleff ZA, Kamper SJ, Maher CG, Evans R, Broderick C, Henschke N. Low back pain in children and adolescents: a systematic review and metaanalysis evaluating the effectiveness of conservative interventions. Eur Spine J. 2014;23:2046-58.

6. Qaseem A1, Wilt TJ, McLean RM, Forciea MA; Clinical Guidelines Committee of the American College of Physicians. Noninvasive treatments for acute, subacute, and chronic low back pain: a clinical practice guideline from the American College of Physicians. Ann Intern Med. 2017; 166: 514-530.

7. Stochkendahl MJ, Kjaer P, Hartvigsen J, Kongsted A, Aaboe J, Andersen M, Andersen M $\varnothing$, Fournier G, Højgaard B, Jensen MB, Jensen LD, Karbo T, Kirkeskov L, Melbye M, Morsel-Carlsen L, Nordsteen J, Palsson TS, Rasti Z, Silbye PF, Steiness MZ, Tarp S, Vaagholt M. National Clinical Guidelines for non-surgical treatment of patients with recent onset low back pain or lumbar radiculopathy. Eur Spine J. 2018;27(1):60-75.

8. Savigny P, Watson P, Underwood M; Guideline Development Group. Early management of persistent non-specific low back pain: summary of NICE guidance. BMJ. 2009; 4;338:b1805.

9. Geneen LJ, Moore RA, Clarke C, Martin D, Colvin LA, Smith BH. Physical activity and exercise for chronic pain in adults: an overview of Cochrane reviews. Cochrane Database Syst Rev. 2017;2017(4):CD011279.

10. Newman-Beinart A, Norton S, Dowling D, Gavriloff D, Vari C, Weinman JA, Godfrey EL. The development and initial psychometric evaluation of a measure assessing adherence to prescribed exercise: the exercise adherence rating scale (EARS). Physiotherapy. 2017;103(2):180-5.

11. Meade LB, Bearne LM, Godfrey EL. Comprehension and face validity of the exercise adherence rating scale in patients with persistent musculoskeletal pain. Musculoskeletal Care. 2018;16(3):409-12. 
12. Guillemin F, Bombardier C, Beaton D. Cross-cultural adaptation of healthrelated quality of life measures: literature review and proposed guidelines. J Clin Epidemiol. 1993;6:1417-32.

13. Beaton DE, Bombardier C, Guillemin F, Ferraz MB. Guidelines for the process of cross-cultural adaptation of self-report measures. Spine (Phila Pa 1976). 2000;25(24):3186-91.

14. Prinsen CAC, Mokkink LB, Bouter LM, Alonso J, Patrick DL, de Vet HCW, Terwee CB. COSMIN guideline for systematic reviews of patient-reported outcome measures. Qual Life Res. 2018;27(5):1147-57.

15. Deyo RA, Dworkin SF, Amtmann D, Andersson G, Borenstein D, Carragee E, Carrino J, Chou R, Cook K, DeLitto A, Goertz C, Khalsa P, Loeser J, Mackey S, Panagis J, Rainville J, Tosteson T, Turk D, Von Korff M, Weiner DK. Focus article: report of the $\mathrm{NIH}$ task force on research standards for chronic low Back pain. Eur Spine J. 2014;23(10):2028-45.

16. Murden RA, McRae TD, Kaner S, Bucknam ME. Mini-mental state exam scores vary with education in blacks and whites. J Am Geriatr Soc. 1991;39: 149-55.

17. Nicholas MK. Self-efficacy and chronic pain. Paper presented at the annual conference British Psychological Society, St. Andrews, Scotland: In; 1989.

18. Jamir Sardá Jr, Nicholas MK, Pimenta CAM, Asghari A. Pain-related selfefficacy beliefs in a Brazilian chronic pain patient sample: a psychometric analysis. Stress and Health. 2007; 23:185-190.

19. Abreu AM, Faria CDCM, Cardoso SMV, Teixeira-Salmela LFT. The Brazilian version of the fear avoidance beliefs questionnaire. Cad Saúde Pública. 2008;24(3):615-23.

20. Sehn F, Chachamovich E, Vidor LP, Dall-Agnol L, de Souza IC, Torres IL, Fregni F, Caumo W. Cross-cultural adaptation and validation of the Brazilian Portuguese version of the pain catastrophizing scale. Pain Med. 2012;13(1): 1425-35.

21. Pais-Ribeiro J, Silva I, Ferreira T, Martins A, Meneses R, Baltar M. Validation study of a Portuguese version of the hospital anxiety and depression scale. Psychol Health Med. 2007;12(2):225-35 quiz 235-7.

22. Nusbaum L, Natour J, Ferraz MB, Goldenberg J. Translation, adaptation and validation of the Roland-Morris questionnaire - Brazil Roland-Morris. Braz J Med Biol Res. 2001;34:203-10.

23. Costa LOP, Maher CG, Latimer J, Ferreira PH, Ferreira ML, Pozzi GC, Freitas LM. Clinimetric testing of three self-report outcome measures for low Back pain patients in Brazil which one is the best? Spine. 2008;33(22):2459-63.

24. Terwee CB, Bot SD, de Boer MR, van der Windt DA, Knol DL, Dekker J, Bouter LM, de Vet HC. Quality criteria were proposed for measurement properties of health status questionnaires. J Clin Epidemiol. 2007;60:34-42.

25. Ostelo RW, Deyo RA, Stratford P, Waddell G, Croft P, Von Korff M, Bouter LM, de Vet HC. Interpreting change scores for pain and functional status in low back pain: towards international consensus regarding minimal important change. Spine (Phila Pa 1976). 2008, 1;33(1):90-4.

26. Mokkink LB, Prinsen CAC, Patrick DL, Alonso J, Bouter LM, de Vet HCW, Terwee CB. COSMIN methodology for systematic reviews of patientreported outcome measures (PROMs) user manual. 2018, version 1.0. https://www.cosmin.nl/wp-content/uploads/COSMIN-syst-review-for-PROMsmanual_version-1_feb-2018.pdf.

27. Clark LA, Watson D. Constructing validity: new developments in creating objective measuring instruments. Psychol Assess. 2019;31(12):1412-27.

28. de Vet HC, Terwee CB, Ostelo RW, Beckerman H, Knol DL, Bouter LM. Minimal changes in health status questionnaires: distinction between minimally detectable change and minimally important change. Health Qual Life Outcomes. 2006; 22;4:54

29. Kamper SJ, Maher CG, Mackay G. Global rating of change scales: a review of strengths and weaknesses and considerations for design. J Man Manip Ther. 2009;17(3):163-70.

30. Fleiss JL, Levin B, Paik MC. Statistical methods for rates and proportions. Hoboken: New Jersey: John Wiley \& Sons. 2003.

31. Weir JP. Quantifying test-retest reliability using the intraclass correlation coefficient and the SEM. J Strength Cond Res. 2005;19:231-40.

32. Zygmont C, Smith MR. Robust factor analysis in the presence of normality violations, missing data, and outliers: empirical questions and possible solutions. The Quantitative Methods for Psychology. 2014;10(1):40-55.

33. Byrne BM. Structural equation modeling with AMOS: basic concepts, applications, and programming. New York, NY: Routledge; 2010.

34. Nevitt J, Hancock GR. Performance of bootstrapping approaches to model test statistics and parameter standard error estimation in structural equation modeling. Struct Equ Model. 2001;8:353-77.
35. Bollen KA, Stine RA. Bootstrapping goodness-of-fit measures in structural equation models. Sociol Methods Res. 1992;21:205-29.

36. Schermelleh-Engel K, Moosbrugger $H$, Müller $H$. Evaluating the fit of structural equation models: test of significance and descriptive goodnessof-fit measures. Methods Psychol Res-Online. 2003;8:23-74.

37. Kaiser HF. The application of eletronic computers to factor analysis. Educational and Psychological Measurement Thousand Oaks. 1960;20:141-51.

38. Dancey CP, Reidy J. Statistics without maths for psychology: using SPSS for windows. New York: Prentice Hall; 2004.

39. Akobeng AK. Understanding diagnostic tests 3: receiver operating characteristic curves. Acta Paediatr. 2007:96(5):644-7.

40. Jack K, McLean SM, Moffett JK, Gardiner E. Barriers to treatment adherence in physiotherapy outpatient clinics: a systematic review. Man Ther. 2010; 15(3):220-8

41. Newman-Beinart A, Goodchild CE, Weinman JA, Ayis S, Godfrey EL. Individual and intervention related factors associated with adherence to home exercise in chronic low back pain: a systematic review. Spine J. 2013; 13(12):1940-50.

42. Picha KJ, Jochimsen KN, Heebner NR, Abt JP, Usher EL, Capilouto G, Uhl TL. Measurements of self-efficacy in musculoskeletal rehabilitation: a systematic review. Musculoskeletal Care. 2018;16(4):471-88.

43. Picha KJ, Lester M, Heebner NR, Abt JP, Usher EL, Capilouto G, Uhl TL. The self-efficacy for home exercise programs scale: development and psychometric properties. J Orthop Sports Phys Ther. 2019:49(9):647-55.

44. Moseley GL, Butler DS. Fifteen years of explaining pain: the past, present, and future. J Pain. 2015;16(9):807-13.

\section{Publisher's Note}

Springer Nature remains neutral with regard to jurisdictional claims in published maps and institutional affiliations.

Ready to submit your research? Choose BMC and benefit from:

- fast, convenient online submission

- thorough peer review by experienced researchers in your field

- rapid publication on acceptance

- support for research data, including large and complex data types

- gold Open Access which fosters wider collaboration and increased citations

- maximum visibility for your research: over $100 \mathrm{M}$ website views per year

At $\mathrm{BMC}$, research is always in progress.

Learn more biomedcentral.com/submissions 